\title{
Study of imbibition in various geometries using phase field method
}

\author{
Junfeng Xiao ${ }^{\circledR}$, Youming Luo, Muyuan Niu, Qiang Wang, Jiali Wu, Xiang Liu, Jianfeng $\mathrm{Xu}^{*}$ \\ State Key Lab of Digital Manufacturing Equipment and Technology, School of Mechanical Science and Engineering, Huazhong University \\ of Science and Technology, Wuhan 430074, P. R. China
}

Keywords:

Imbibition

phase field

capillary pressure

geometry

Cited as:

Xiao, J., Luo, Y., Niu, M., Wang, Q., Wu, J., Liu, X., Xu, J. Study of imbibition in various geometries using phase field method. Capillarity, 2019, 2(4): 57-65, doi: 10.26804/capi.2019.04.01.

\begin{abstract}
:
Phase field method has been widely utilized to study multiphase flow problems, but has seldom been applied to the study of imbibition. Previous methods used to simulate imbibition, such as moving mesh method, need to specify capillary pressure as a boundary condition a priori, whereas phase field method can calculate capillary pressure automatically for various geometries. Therefore, phase field method would be a versatile tool for the study of imbibition in various geometries. In this paper, phase field method is employed to solve dynamical imbibition problem in various geometries, including straight tube, conical tube and structures in which the topology changes. The variation of the imbibition height with respect to time from phase field simulation is verified with theoretical predictions from Lucas-Washburn law in a straight capillary tube with three gravitational scenarios. In addition, the capillary pressure and velocity field are found to be consistent with Laplace-Young equation and Hagen-Poiseuille equation in various geometries. The applicability and accuracy of the phase field method for the study of imbibition in structures with changing topology are also discussed.
\end{abstract}

\section{Introduction}

Imbibition is a spontaneous flow behavior driven by capillary pressure, which means no additional energy input is needed to propel the flow. Imbibition is ubiquitous in nature, for example, it is crucial for transportation of water from soil to leaves in plants (Wheeler and Stroock, 2008). On the other hand, people have utilized imbibition to assistant the extraction of underground oil, and to predict liquid transport in the printing and textile industries. Recently, researchers have developed paper-based microfluidic devices, a cheap and portable alternative to conventional microfluidic chip, in which imbibition is the sole driving force of liquid flow and therefore pumps are no longer required (Osborn et al., 2010; Mehrabian et al., 2011; Cate et al., 2015). Therefore, given its scientific and engineering significance, a better understanding of imbibition is warranted.

The driving force of imbibition, i.e., capillary pressure, is determined by interfacial properties (interfacial tension, contact angle, and geometry of the media). The well-known Lucas-Washburn equation indicates that the wetted length $l$ increases as a function of time $t$ in the form $l \sim t^{1 / 2}$, which is appropriate for one-dimensional imbibition (Washburn, 1921). As the geometry expands from one dimension to two or three dimensions (Xiao et al., 2012), different time exponents have been discovered: $1 / 4,1 / 3,0.46$, etc. At the same time, imbibition is a dynamical process, and it is also influenced by inertia and body force such as gravity (Fries and Dreyer, 2009; Masoodi et al., 2013).

The effects of geometry on imbibition invite investigations from different aspects. When a channel is not uniform, there exists an optimum combination of geometrical parameters that provides the fastest imbibition (Shou et al., 2014a, 2014b). Also, in the presence of two branches of channels, preferred imbibition in one of the channels has been found (Mehrabian et al., 2011; Sadjadi et al., 2015). Fractal theory has also been used to characterize the interconnected networks of porous media (Cai and Yu, 2011; Cai et al., 2012). For complex geometries, analytical solutions are extremely scarce, so numerical simulation is an important approach for the study of imbibition.

One special aspect of simulations of imbibition is that the wetted region evolves, and consequent computational domain changes. Moving mesh method has been adopted to account

${ }^{*}$ Corresponding author.

E-mail address: xiaojf@hust.edu.cn (J. Xiao); youmingluo@hust.edu.cn (Y. Luo); muyuan@hust.edu.cn (M. Niu); qiang_w@hust.edu.cn (Q. Wang); wujiali@hust.edu.cn (J. Wu); liu_xiang@hust.edu.cn (X. Liu); jfxu@hust.edu.cn (J. Xu). 2652-3310 (C) The Author(s) 2019.

Received October 12, 2019; revised October 21, 2019; accepted October 22, 2019; available online October 25, 2019. 
for evolving domains (Xiao et al., 2018), but capillary pressure has to be known a priori, which is not appropriate for complex geometries. Interface tracking methods such as phase field method, level set method and volume-of-fluid method provide powerful tools for different geometries. Level set method (Bashir et al., 2011; Akhlaghi Amiri and Hamouda, 2013) is not as accurate as phase field method in terms of pressure gradients and velocity profiles, and phase field method is more successful in capturing the physical details especially in complicated porous media. Moreover, level set method has the problem of volume conservation. On the other hand, it is difficult for volume-of-fluid method (Liu and $\mathrm{Yu}, 2016$ ) to describe complex geometry on the interface and define the interfacial tension, while the evolution of a complicated free surface can be naturally followed without any special consideration for phase field method. Although phase field method is generally used for multiphase problems (Jacqmin, 1999; Yue et al., 2004; Qin and Bhadeshia, 2010; Akhlaghi Amiri and Hamouda, 2013; Bai et al., 2017), it will be shown in this paper that phase field method can be utilized to accurately solve imbibition problems and can readily accommodate various geometries. Through this research, it will be demonstrated that phase field method is a powerful tool in the study of imbibition in complex geometries.

\section{Theory and model}

The phase field method combines Navier-Stokes equation with Cahn-Hilliard diffusion equation (Badalassi et al., 2003; Qin and Bhadeshia, 2010; Zhou et al., 2010; Bai et al., 2017; Rokhforouz and Akhlaghi Amiri, 2017):

$$
\rho \frac{\partial \mathbf{u}}{\partial t}+\rho(\mathbf{u} \cdot \nabla) \mathbf{u}=\nabla \cdot\left[-p \mathbf{I}+\mu\left(\nabla \mathbf{u}+\nabla \mathbf{u}^{\mathrm{T}}\right)\right]+\mathbf{F}_{\mathrm{st}}+\mathbf{F}_{\mathrm{ext}}+\mathbf{F}_{\mathrm{g}}
$$

$$
\nabla \cdot \mathbf{u}=0
$$

$$
\begin{gathered}
\frac{\partial \phi}{\partial t}+\mathbf{u} \cdot \nabla \phi=\nabla \cdot \frac{\gamma \lambda}{\varepsilon^{2}} \nabla \psi \\
\psi=-\nabla \cdot \varepsilon^{2} \nabla \phi+\left(\phi^{2}-1\right) \phi+\left(\frac{\varepsilon^{2}}{\lambda}\right) \frac{\partial f_{\mathrm{ext}}}{\partial \phi}
\end{gathered}
$$

where $p$ is the pressure, $\mathbf{u}$ is the fluid velocity field, $t$ is time, $\mu$ is the viscosity of liquid, $\gamma$ is the mobility parameter, $\psi$ is an auxiliary variable (Qin and Bhadeshia, 2010) that decomposes a fourth-order equation into two second-order equations, $\lambda$ is the mixing energy density, and $\varepsilon$ is a control parameter for the interface thickness that scales with thickness of the interface. The parameters $\lambda$ and $\varepsilon$ are related to interfacial tension $\sigma$ through the equation:

$$
\sigma=\frac{2 \sqrt{2}}{3} \frac{\lambda}{\varepsilon}
$$

where $\phi$ is the phase variable, which varies in $[-1,1]: \phi=1$ in the pure liquid phase and -1 in the pure gas phase, while it changes in $(-1,1)$ at the gas-liquid mixing zone.
The interfacial tension in phase field method is implemented as a body force:

$$
\mathbf{F}_{\mathrm{st}}=\left(G-\frac{\partial f}{\partial \phi}\right) \nabla \phi
$$

where $G$ is the chemical potential, the value of which is calculated according to the formula:

$$
G=\lambda\left[-\nabla^{2} \phi+\frac{\phi\left(\phi^{2}-1\right)}{\varepsilon^{2}}\right]+\frac{\partial f}{\partial \phi}
$$

where $\partial f / \partial \phi$ is a user-defined source of free energy. The source of free energy is taken as 0 in this work. $\mathbf{F}_{\mathrm{g}}$ is the body force exerted on the fluids, such as gravity. $\mathbf{F}_{\text {ext }}$ is an external force due to the user-defined source of free energy, and $\mathbf{F}_{\text {ext }}=0$ in this work. The specific theory and details of phase field method have been widely discussed (Jacqmin, 1999; Qin and Bhadeshia, 2010; Fakhari et al., 2018), and will not be elaborated in this paper.

In the phase field model, the interfacial thickness $\varepsilon$ and mobility $\gamma$ are two particularly important parameters. Too small an interfacial thickness $\varepsilon$ will lead to a great increase in computing cost and cause difficulties in convergence with the phase field method, and too large a value of $\varepsilon$ will increase the numerical error. The value of $\varepsilon$ is half of the maximum mesh size in this study. The mobility parameter $\gamma$ determines the time scale of the Cahn-Hilliard diffusion, and it thereby governs the diffusion-related time scale for the interface. Too small a mobility parameter may lead to distortion of the meniscus. The specific value of the mobility parameter usually needs to be determined in combination with specific experiments or existing theories.

The study of effect of mobility parameters is illustrated in Fig. S in Appendix material. Different mobility parameters result in different rise height for one dimensional imbibition. When the mobility parameter $\gamma$ is $0.2 \mathrm{~m} \cdot \mathrm{s} / \mathrm{kg}$, the results from simulation agree well with the theoretical results. However, when the mobility parameter is less than 0.2 , the imbibition height from phase field method obviously lags behind the theoretical curve; when the mobility parameter is 1 , the imbibition height from phase field method will be ahead of the theoretical value. It can also be seen from Figs. S(b)-(d) that when the mobility parameter is different, there will be obvious difference in the curvature of the meniscus. When the mobility parameter $\gamma$ is $2 \times 10^{-4} \mathrm{~m} \cdot \mathrm{s} / \mathrm{kg}$, the interface is flat, and the curvature of the meniscus is infinite, which is contrary to the setting of contact angle $(\theta=\pi / 6)$. When the mobility parameter $\gamma$ is 1 or larger, the curvature of the meniscus would be too small and the imbibition velocity would be too fast. When the mobility parameter $\gamma$ is $0.2 \mathrm{~m} \cdot \mathrm{s} / \mathrm{kg}$, the result from phase field method is in line with theory from Washburn (1921). Therefore, $\gamma=0.2 \mathrm{~m} \cdot \mathrm{s} / \mathrm{kg}$ is selected, and it is applied for other geometries in this paper.

In this work, commercial finite element software COMSOL Multiphysics is employed to solve the phase field model. For the boundary conditions in phase field model, the pressure at the source is set as atmospheric pressure $p_{0}$, as is the pressure at the exit. The pressure at the wetting front doesn't need 
Table 1. Parameters of simulation.

\begin{tabular}{lll}
\hline Parameters & Values & Description \\
\hline$\rho$ & $1000 \mathrm{~kg} / \mathrm{m}^{3}$ & Density of silicone oil \\
$\mu$ & $0.1 \mathrm{~Pa} \cdot \mathrm{s}$ & Viscosity of silicone oil \\
$\sigma$ & $0.03 \mathrm{~N} / \mathrm{m}$ & Interfacial tension \\
$\theta$ & $\pi / 6$ & Contact angle \\
$r$ & $0.1 \mathrm{~mm}$ & Radius of straight capillary tube \\
$\gamma$ & $0.2 \mathrm{~m} \cdot \mathrm{s} / \mathrm{kg}$ & Mobility parameter \\
$h$ & $0.02 \mathrm{~mm}$ & The maximum mesh size \\
$\varepsilon$ & $h / 2$ & Control parameter for the interface thickness \\
$\alpha$ & $4^{\circ}$ & Opening angle of expended conical tube \\
$r_{0}$ & $0.1 \mathrm{~mm}$ & Initial radius of conical tube \\
\hline
\end{tabular}
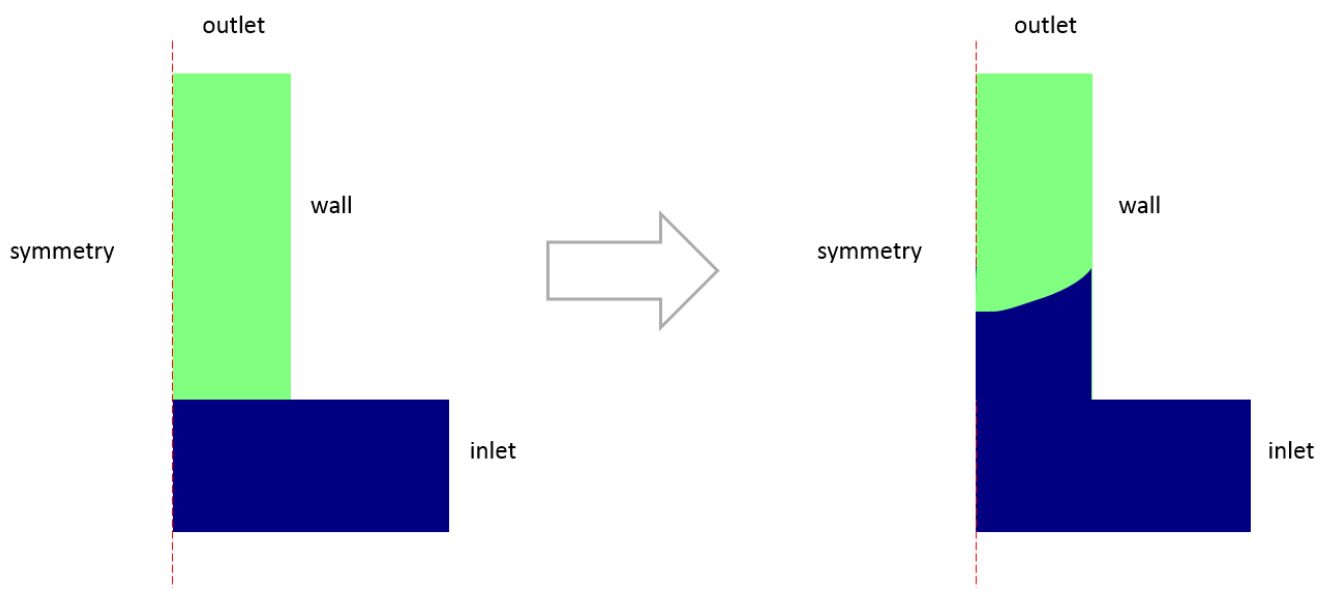

Fig. 1. Two-dimensional axisymmetric model for the imbibition process.

to be set a priori, since it can be calculated automatically using phase field model, which replicates the real imbibition process.

Fluid properties have a great influence on imbibition height. The parameters of the fluid and tube in the simulation are taken as follows: Density $\rho=1 \times 10^{3} \mathrm{~kg} / \mathrm{m}^{3}$, viscosity $\mu=0.1 \mathrm{~Pa} \cdot \mathrm{s}$, interfacial tension $\sigma=0.03 \mathrm{~N} / \mathrm{m}$, contact angle $\theta=\pi / 6$, and radius $r=0.1 \mathrm{~mm}$. The specific parameters in simulations are listed in Table 1.

Fig. 1 shows the geometry of the simulated domain. The two-dimensional axisymmetric model is used, with the blue area filled with liquid and the gray area with air initially. Usually, the value of $\gamma$ is determined experimentally, in this work, $\gamma$ is $0.2 \mathrm{~m} \cdot \mathrm{s} / \mathrm{kg}$. Hydrodynamic mesh calibration and a structured mapping mesh are used, and the maximum mesh size $h$ is taken as $0.02 \mathrm{~mm}$. The specific settings of boundary conditions are shown in Table 2.

\section{Results and discussion}

In order to verify the accuracy of the phase field method, the imbibition results are analyzed under three imbibition con-
Table 2. Boundary conditions for simulations.

\begin{tabular}{ll}
\hline Boundary & Condition \\
\hline Inlet & Pressure $=$ atmospheric pressure \\
Outlet & Pressure $=$ atmospheric pressure \\
Wall & Velocity: no slip \\
Axis of symmetry & Axis of symmetry \\
\hline
\end{tabular}

ditions in a straight capillary tube: Gravity-free, gravity induced acceleration, and gravity induced deceleration, and the capillary pressure is examined in detail.

Fig. 2 shows the imbibition height with respect to time. When neglecting gravity, the result of simulation is in good agreement with the classic Lucas-Washburn law at large time $t$. In the classic Lucas-Washburn law, the Laplace-Young pressure is assumed to be a constant, which means the wetting front assumes the shape of a meniscus after the initial stage. However, there is a transitional period for the wetting front during which it changes from a flat shape to a meniscus. Phase 


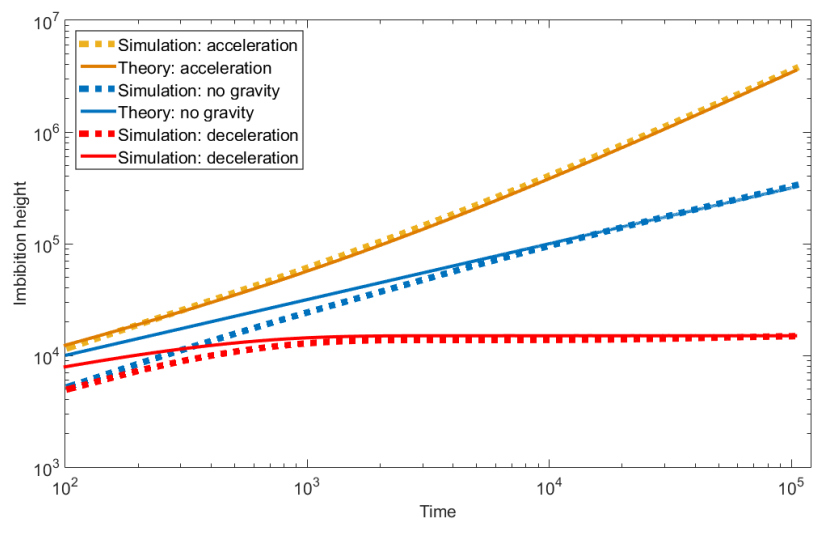

Fig. 2. Comparison of imbibition results in a straight tube without gravity, with gravity acting as an accelerator, and with gravity acting as a decelerator. The results in the three scenarios are shown in terms of dimensionless time $T=2 P_{c} t / \mu$ and imbibition height $L=2 \sqrt{2} l / r$.

field simulation is able to capture the transition and the consequent increase in capillary pressure. Therefore, imbibition from theoretic prediction is faster than that from simulation in the initial period. After this transitional period, it can be seen that the simulation results are consistent with the theoretical predictions, which will also be demonstrated by a subsequent pressure analysis.

There has been much theoretical research on imbibition under the influence of gravity. It has been found that under different effects of gravity, the relationship between imbibition height and time exhibits different exponents. The phase field method is also used to simulate the imbibition phenomenon under different gravitational effects. Fig. 2 shows the dynamical change of imbibition height when gravity acts as a decelerator or an accelerator. When the dynamical effect of the meniscus is ignored in the early stage, it can be seen that the results from phase field method are consistent with theoretical predictions (Xiao et al., 2018).

\subsection{Verification of pressure}

The capillary pressure due to interfacial tension is the driving force of the imbibition phenomenon. Therefore, capillary pressure has an important influence on imbibition, which can be calculated using Laplace-Young equation:

$$
P_{c}=\frac{2 \sigma \cos \theta}{r}
$$

According to Eq. (8), the theoretical value of capillary pressure is $519.6152 \mathrm{~Pa}$ when gravity is ignored. The capillary pressure is three orders of magnitude smaller than atmospheric pressure, and consequently the pressure changes are small. In order to better observe the change of pressure, atmospheric pressure is subtracted from the interfacial pressure, and the resulting relationship between pressure and time is described below.

Fig. 3 shows the dynamical changes in capillary pressure with time from phase field model. In the very short time of the initial period, the liquid surface transforms from a flat shape to a meniscus, with a consequent change in its curvature. This

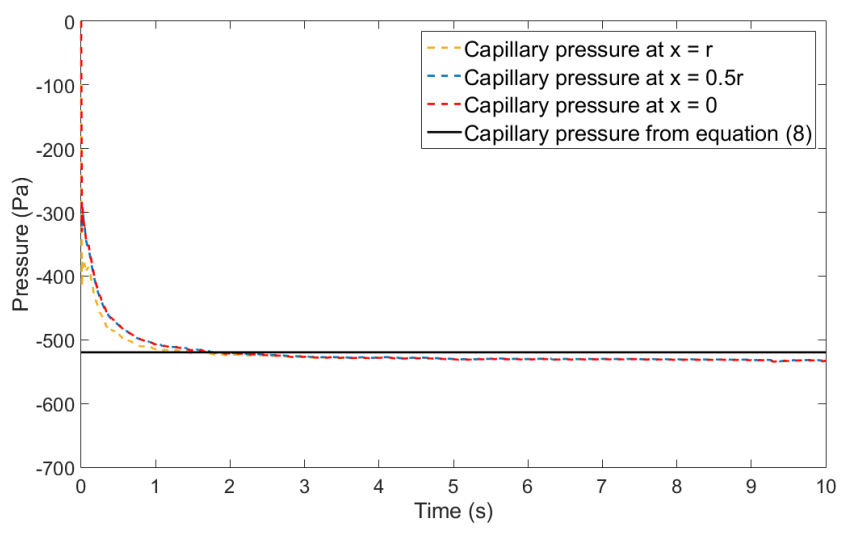

Fig. 3. Dynamical changes in capillary pressure with time in phase field simulation. By calculating the minimum pressure at different radii, the capillary pressure at the interface is captured.

change in shape of the interface is called the entrance effect (Blake and Coninck, 2004; Hultmark et al., 2011). The entrance effect is neglected in classic Lucas-Washburn theory, which leads to a discrepancy between the capillary pressure calculated by phase field model and theoretical capillary pressure in the initial stage. As can be seen from Fig. 3, the capillary pressure from phase field model converges to theoretical capillary pressure at long times. Therefore, the phase field model is reliable in calculating capillary pressure and is capable of capturing the precise changes in capillary pressure in the transitional stage.

\subsection{Verification of velocity}

From Eq. (2), it can be seen that the divergence of velocity is zero in the process of imbibition. Therefore, the following equation holds:

$$
\frac{\partial u}{\partial x}+\frac{\partial v}{\partial y}+\frac{\partial w}{\partial z}=0
$$

where $u, v$, and $w$ are the velocity components in the $x, y$, and $z$ directions, respectively. In the axisymmetric model, since there are no velocity components in the $x$ and $y$ directions, Eq. (9) simplifies to:

$$
\frac{\partial w}{\partial z}=0
$$

which means velocity must be uniform in the vertical direction. The velocity of imbibition is affected by fluid properties and tube geometry. According to Lucas-Washburn theory, the relationship between the imbibition velocity in the vertical direction and time can be obtained by taking the derivative of the rise height, with the following result:

$$
v=\frac{1}{2}\left(\frac{r \sigma \cos \theta}{2 \mu}\right)^{\frac{1}{2}} t^{-\frac{1}{2}}
$$

Fig. 4 shows the velocity changes during imbibition. It can be seen from Fig. 4(a) that the velocity decreases with time, and the velocity obtained from the simulation model is consistent with the theoretical velocity at any time. Fig. 4(b) shows the variation of velocity distribution with height in a 

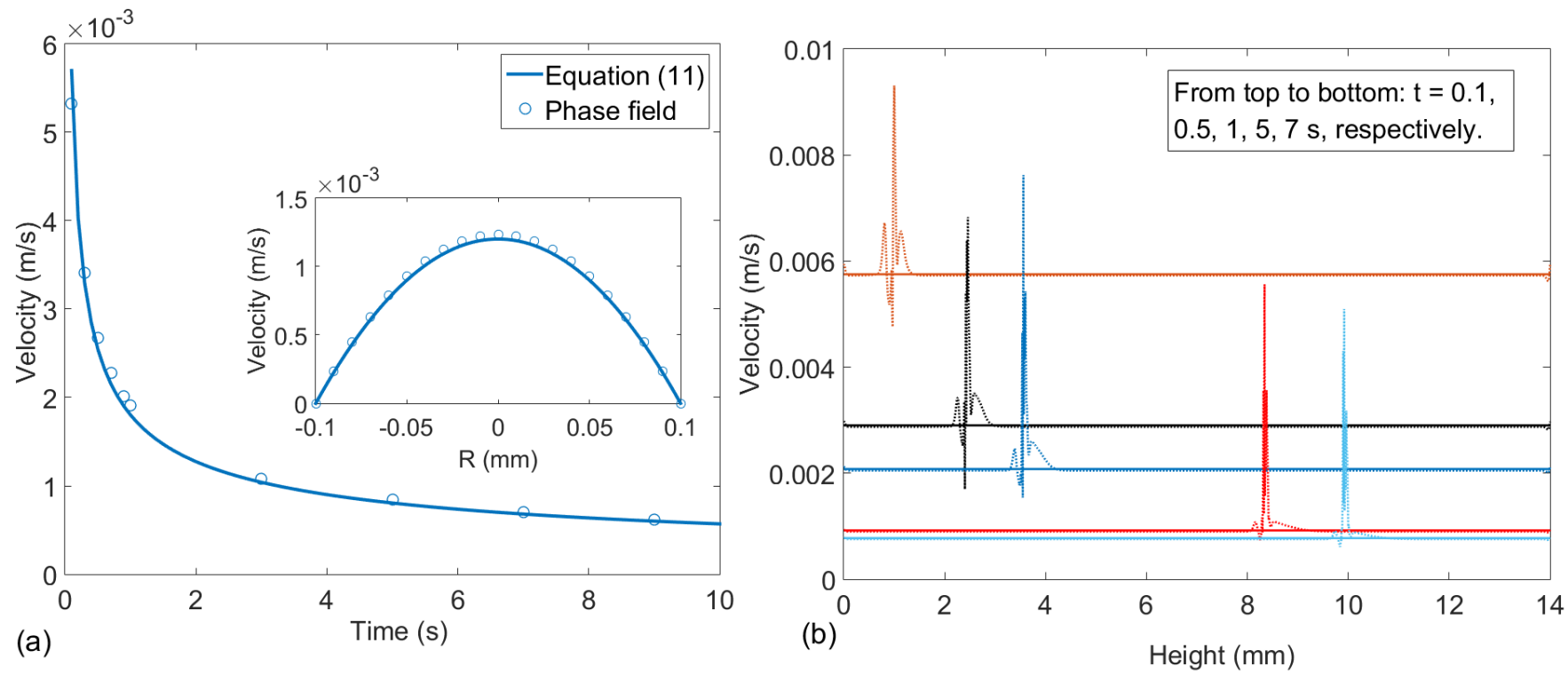

Fig. 4. (a) Comparison of simulated and theoretical velocities at $x=0.707 r$. The circles represent the result derived from the phase field method, and the curve shows Hagen-Poiseuille velocity distribution according to Eq. (11). The inset shows the velocity distribution on the meniscus at $t=7 \mathrm{~s}$. (b) vertical velocity distribution in a straight tube at $t=0.1,0.5,1,5$, and $7 \mathrm{~s}$ (curves from top to bottom). The solid line represents the average velocity, while the dashed line represents the actual velocity distribution in the capillary tube.

straight tube at different times. There is a small step region on the velocity curves, which is caused by the thickness of the interface in the phase field model. The complex flow state in the liquidair mixing region leads to the oscillations of velocity, which will not be discussed further in this paper. Focusing on the liquid phase in Fig. 4(b), it can be seen that the velocity does not change in vertical direction, which is consistent with Eq. (10). It further verifies the accuracy of the phase field model.

The velocity distribution on the meniscus will affect the accuracy of the imbibition flow, so it needs to be verified. The inset of Fig. 4(a) shows the velocity distribution on the meniscus at $t=7 \mathrm{~s}$. It can be seen that velocity distribution conforms to the velocity distribution of fully developed laminar flow, i.e., a parabolic profile, which illustrates the universality of the phase field method from the aspect of velocity distribution.

Mass conservation is one of the conditions that should be verified for phase field method. Since silicone oil is assumed to have an infinite supply in this simulation model, it is difficult to directly verify the dynamic mass conservation. Therefore, conservation of mass can be indirectly proved by verifying the conservation of flow. Because the capillary tube section is uniform, the flow is conserved as long as the velocity is uniform according to Eq. (10). It can be seen from Fig. 4(b) that the velocity distribution is constant except for the velocity fluctuations at the interface (the meniscus). Therefore, if the average velocity at the fluctuation is consistent with the velocity distribution curve, the model satisfies mass conservation. Fig. 4(b) shows the average velocity curve near the interface and the overall velocity distribution at different times. As can be seen from the figure, the average velocity is consistent with the distribution of the overall velocity distribution at any given moment, which indirectly indicates that the model satisfies the conservation of mass.

\subsection{Effects of geometry}

In the above work, the phase field method has been used to analyze the imbibition in a straight tube. By comparing with Lucas-Washburn law and other classical theories, the reliability of the phase field method has been verified through the capillary pressure and the velocity distribution. However, most imbibition occurs in more complex geometries, where expansion and bifurcation are common. In this subsection, capillary flow in conical tubes and bifurcated-coalescent tubes will be studied using phase field method.

The dynamical changes of imbibition flow in conical tubes have been studied from the perspective of both theory and experiment (Reyssat et al., 2008; Berli and Urteaga, 2014). In an expanded conical tube, the curvature of the wetting front is constantly changing, which means capillary pressure changes with meniscus position. When phase field method is used to simulate imbibition in an expanded conical tube, the capillary pressure need not be set a priori, and it can be calculated automatically in the simulation. The boundary conditions are same to that in straight tube illustrated in Fig. 1 and Table 2. In this discussion, the effect of gravity will be ignored, the opening angle of the expanded conical tube will be taken as $4^{\circ}$, and the initial inner radius $r_{0}$ as $0.1 \mathrm{~mm}$, while the other parameters are the same as those for the straight tube.

Fig. 5 shows the relation between the imbibition height and time in an expanded conical tube. The full curve represents the theoretical results of Reyssat et al. (2008) and the dashed curve the variation of imbibition height with time obtained by simulation using phase field method. It can be seen that the simulation results are consistent with the theoretical derivation.

In a conical tube, as the meniscus moves forward, the inner diameter increases, which leads to a change in the curvature 


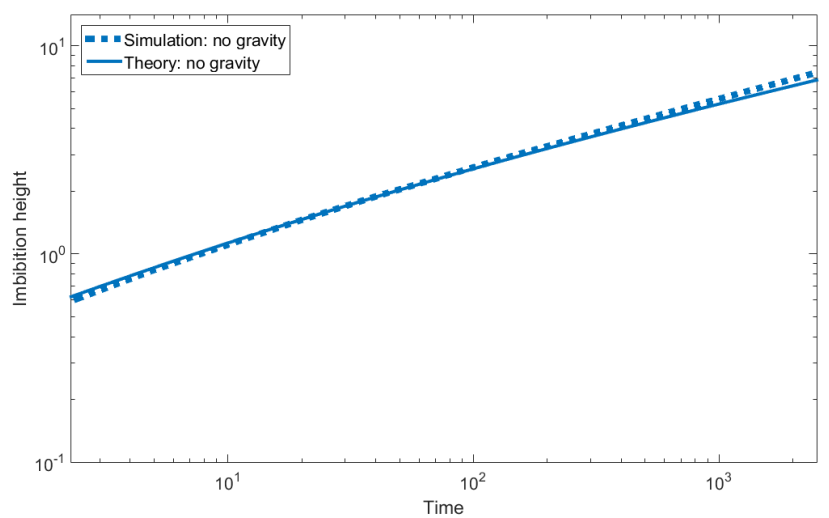

Fig. 5. Dimensionless plot of imbibition height vs time for imbibition in an expanding conical tube. Time and imbibition height are nondimensionalized as $T=2 \sigma \alpha^{2} t \cos (\theta+\alpha) /\left(\mu r_{0}\right)$ and $L=\alpha l / r_{0}$, respectively.

of the wetting front. Therefore, the capillary pressure does not remain constant. It is therefore crucial to verify the relationship between the capillary pressure and the position of the meniscus.

According to theoretical derivation, the capillary pressure in a conical tube is given by:

$$
p_{c}=\frac{2 \sigma \cos (\theta+\alpha)}{R}
$$

where $R$ is the inner diameter of the conical tube corresponding to the imbibition height $l$, and is related to the latter by:

$$
R=r_{0}+l \alpha
$$

Combining Eqs. (12) and (13) gives the following relationship between capillary pressure $p_{c}$ and imbibition height $l$ :

$$
p_{c}=\frac{2 \sigma \cos (\theta+\alpha)}{r_{0}+l \alpha}
$$

Fig. 6 shows the variation of capillary pressure with meniscus position in a conical tube. As can be seen, when the imbibition height $l<1 \mathrm{~mm}$, there is a discrepancy between the theoretical results and those from the simulation. This is because in the phase field model, the wetting front is converted from the initial flat surface to a meniscus, which means capillary pressure takes a certain time to change from a very small value to a normal capillary pressure. However, in the theoretical analysis, the meniscus dynamics is not taken into account, and it is assumed that the capillary pressure remains constant as the imbibition height increases. Therefore, there is a discrepancy between the theoretical and simulated pressures when the imbibition height is small. At large imbibition times, the theoretical and simulated capillary pressure converge on each other, which indicates the universality of the phase field method for imbibition in complex geometric structures.

Topological changes, such as bifurcation and coalescence, are another very important aspect of geometry in the imbibition media. For example, the imbibition in the leaves of plants can be seen as involving a complex structure composed of numerous bifurcated-coalescent tubes. Therefore, study of

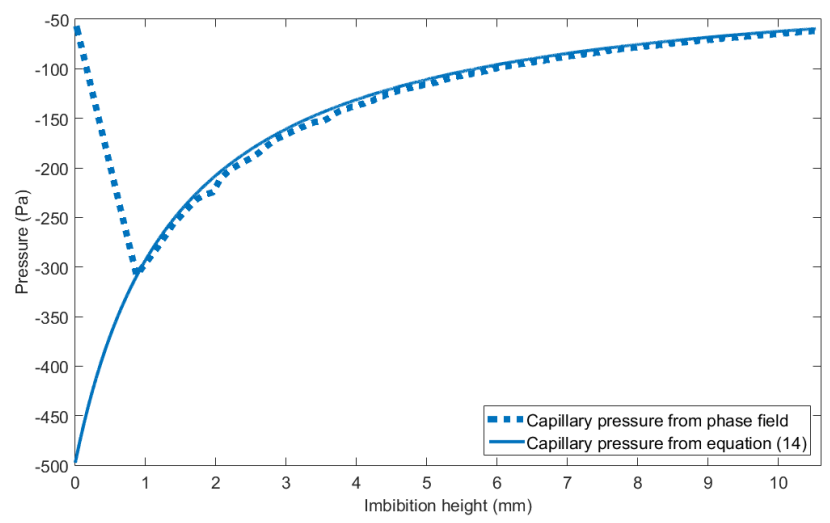

Fig. 6. Variation of capillary pressure with imbibition height. The solid curve represents the theoretical variation according to Eq. (14) and the dotted curve the variation according to the phase field model.

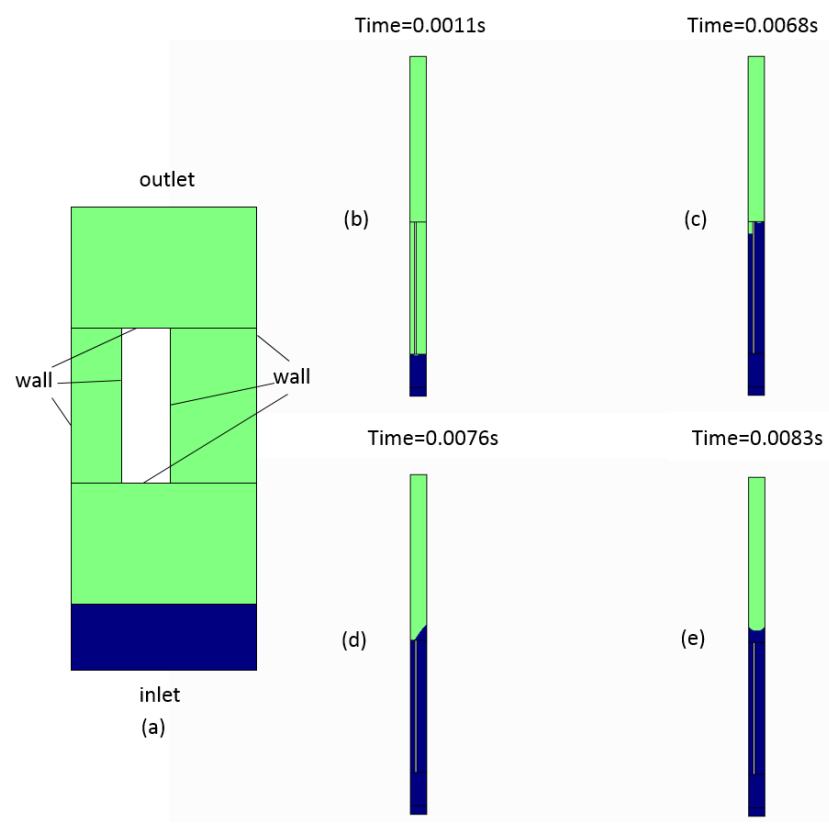

Fig. 7. Schematic of complex topological structure and the rise height of the meniscus at different times. (a) Model of bifurcated-coalescent tube. (b) The meniscus breaks into two small menisci at the bifurcation. (c) Imbibition in the wide tube eventually becomes faster than that in the narrow tube. (d) A single meniscus is reconstructed at the terminus of the bifurcation. (e) Imbibition continues in the coalescent tube after reconstruction of the meniscus.

imbibition in such structures is necessary for understanding transportation of liquids in complex geometries.

Fig. 7(a) shows a bifurcated-coalescent structure. Boundary conditions are similar to that in straight tube and expanded tube. Accurate theoretical study of imbibition in this kind of capillary tube is difficult because of the occurrence of meniscus splitting and reconstruction. However, it is much easier to study such complex geometric structures using the phase field method, and the detailed movement of meniscus can be observed, which can provide guidance for theoretical derivations.

Figs. 7(b)-7(e) show simulation results of capillary phenomena in a bifurcated-coalescent tube using phase field method. The tube has a small common area before bifurcation, 


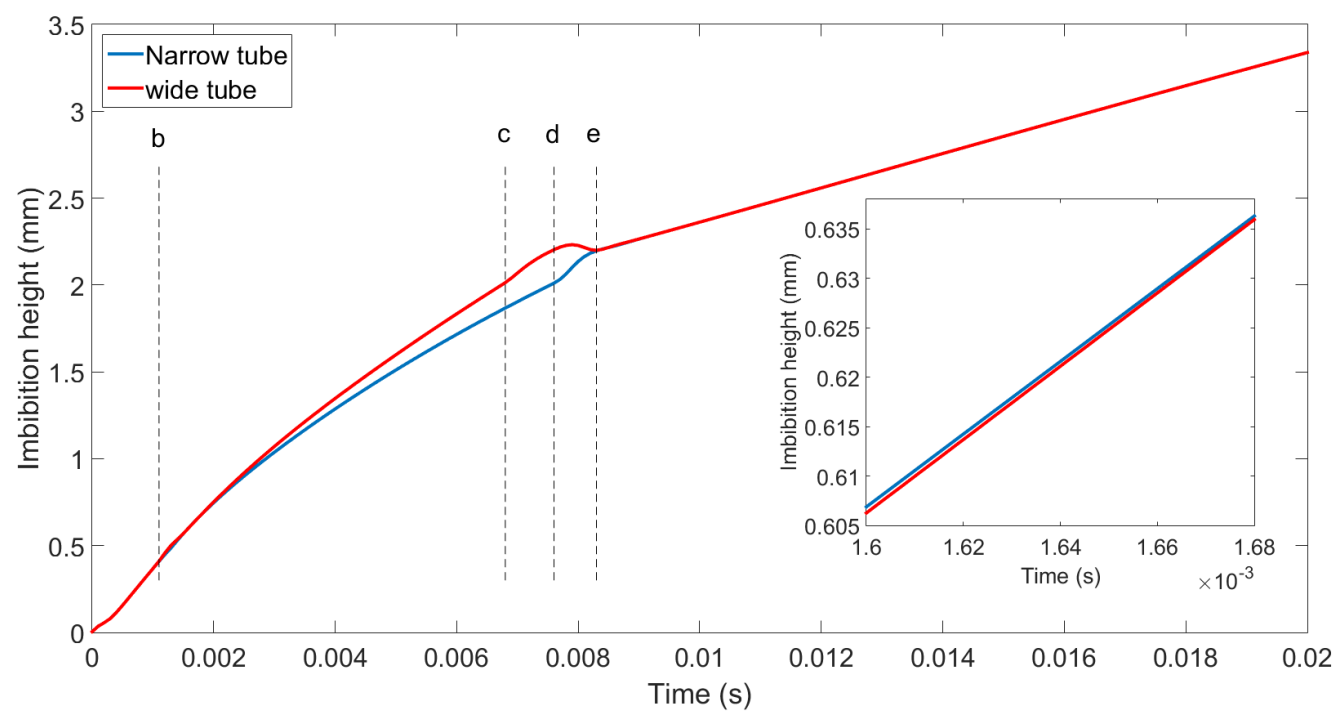

Fig. 8. Imbibition height vs time in a bifurcated-coalescent tube: The blue curve is the imbibition height in the narrow tube and the red curve that in the wide tube. The inset shows that imbibition is faster in the narrow tube for a very short initial period. The vertical dashed lines correspond to different states in Figs. 7(b)-7(e).

which is called the root tube. When the wetting front reaches the peak of the root tube, the meniscus splits into two small menisci, which enter the wide and narrow tubes, respectively. For a very small period of time after entering the bifurcation, the wetting front rises faster in the narrow tube. But after a long period of time, it rises faster in the wide tube than in the narrow one, as shown in Fig. 7(c). This is consistent with theories from literature (Sadjadi et al., 2015) which describes the imbibition in a bifurcated capillary tube in experiments. When the meniscus in the narrow capillary reaches the terminus of the bifurcation, reconstruction of a single meniscus commences (Fig. 7(d)). In this regime, the menisci are pinned, and the meniscus in the narrow tube merges with that in the wide tube to re-form a single large meniscus. When the reconstruction of the meniscus is complete (Fig. 7(e)), it begins to rise again.

As shown in Fig. 8, for a very short period of time after the menisci enter the bifurcated structure, the meniscus in the narrow tube rises faster, because the capillary pressure in the narrow tube is greater, resulting in faster capillary rise, and capillary pressure initially plays the dominant role. However, after about $0.0017 \mathrm{~s}$, the capillary rises faster in the wide tube. This is because viscous dissipation has a more significant effect with increasing rise height and decreasing rise speed, and it also has a larger effect in that narrow tube than in the wide one. Thus, the wide tube has faster imbibition at later times (Tsunazawa et al., 2016).

\section{Conclusion}

In this paper, phase field method has been used to simulate the imbibition in various geometries, including those with topology change. The relationship of imbibition height to time, the dynamical effect of capillary pressure, and the imbibition velocity have been analyzed systematically, and the results from phase field method have been shown to be consistent with the theoretical predictions, which proves that phase field method is an accurate and versatile tool for the study of imbibition. Compared with moving mesh method, phase field method can readily accommodate imbibition in geometries with complex topology, and there is no need to set capillary pressure as a boundary condition a priori. Previous studies of imbibition didn't examine capillary pressure and imbibition velocity, which are the two major factors affecting imbibition, whereas here the capillary pressure and imbibition velocity from phase field simulation are verified with Laplace-Young equation and Hagen-Poiseuille equation. The entrance effect, which represents the transition of the wetting front from a flat shape to a meniscus, is clearly revealed by the phase field method, although it was neglected in previous studies of imbibition. Through this research, the phase field method is proved to be a powerful tool to study imbibition in geometries with complex topology, and it is deemed to be an effective tool to study the transport phenomena in paper based microfluidic device.

\section{Acknowledgement}

This work is supported by National Natural Science Foundation of China (Grant No. 51705172) and funding from State Key Lab of Digital Manufacturing Equipment and Technology.

\section{Conflict of interest}

The authors declare no competing interest.

Open Access This article is distributed under the terms and conditions of the Creative Commons Attribution (CC BY-NC-ND) license, which permits unrestricted use, distribution, and reproduction in any medium, provided the original work is properly cited. 


\section{References}

Amiri, H.A.A., Hamouda, A.A. Evaluation of level set and phase field methods in modeling two phase flow with viscosity contrast through dual-permeability porous medium. Int. J. Multiphas. Flow 2013, 52: 22-34.

Badalassi, V.E., Ceniceros, H.D., Banerjee, S. Computation of multiphase systems with phase field models. J. Comput. Phys. 2003, 190(2): 371-397.

Bai, F., He, X., Yang, X., et al. Three dimensional phasefield investigation of droplet formation in microfluidic flow focusing devices with experimental validation. Int. J. Multiphas. Flow 2017, 93: 130-141.

Bashir, S., Rees, J.M., Zimmerman, W.B. Simulations of microfluidic droplet formation using the two-phase level set method. Chem. Eng. Sci. 2011, 66(20): 4733-4741.

Berli, C.L.A., Urteaga, R. Asymmetric capillary filling of non-Newtonian power law fluids. Microfluid. Nanofluid. 2014, 17(6): 1079-1084.

Blake, T.D., Coninck, J.D. The influence of pore wettability on the dynamics of imbibition and drainage. Colloids Surf. A 2004, 250(1-3): 395-402.

Cai, J., Hu, X., Standnes, D.C., et al. An analytical model for spontaneous imbibition in fractal porous media including gravity. Colloids Surf. A 2012, 414: 228-233.

Cai, J., Yu, B. A discussion of the effect of tortuosity on the capillary imbibition in porous media. Transp. Porous Med. 2011, 89(2): 251-263.

Cate, D.M., Adkins, J.A., Mettakoonpitak, J., et al. Recent developments in paper-based microfluidic devices. Anal. Chem. 2015, 87(1): 19-41.

Fakhari, A., Li, Y., Bolster, D., et al. A phase-field lattice Boltzmann model for simulating multiphase flows in porous media: Application and comparison to experiments of $\mathrm{CO}_{2}$ sequestration at pore scale. Adv. Water Resour. 2018, 114: 119-134.

Fries, N., Dreyer, M. Dimensionless scaling methods for capillary rise. J. Colloid Interf. Sci. 2009, 338(2): 514518.

Hultmark, M., Aristoff, J.M., Stone, H.A. The influence of the gas phase on liquid imbibition in capillary tubes. J. Fluid Mech. 2011, 678: 600-606.

Jacqmin, D. Calculation of two-phase Navier-Stokes flows using phase-field modeling. J. Comput. Phys. 1999, 155(1): 96-127.

Liu, Y., Yu, X. A coupled phasefield and volume-of-fluid method for accurate representation of limiting water wave deformation. J. Comput. Phys. 2016, 321: 459-475.

Masoodi, R., Languri, E., Ostadhossein, A. Dynamics of liquid rise in a vertical capillary tube. J. Colloid Interf. Sci. 2013, 389(1): 268-272.

Mehrabian, H., Gao, P., Feng, J.J. Wicking flow through microchannels. Phys. Fluids 2011, 23(12): 122108.

Osborn, J.L., Lutz, B., Fu, E., et al. Microfluidics without pumps: Reinventing the T-sensor and $\mathrm{H}$-filter in paper networks. Lab Chip 2010, 10(20): 2659-2665.

Qin, R.S., Bhadeshia, H.K. Phase field method. Mater. Sci. Tech-lond. 2010, 26(7): 803-811.

Reyssat, M., Courbin, L., Reyssat, E., et al. Imbibition in geometries with axial variations. J. Fluid Mech. 2008, 615: 335-344.

Rokhforouz, M.R., Akhlaghi Amiri, H.A. Phase-field simulation of counter-current spontaneous imbibition in a fractured heterogeneous porous medium. Phys. Fluids 2017, 29(6): 062104.

Sadjadi, Z., Jung, M., Seemann, R., et al. Meniscus arrest during capillary rise in asymmetric microfluidic pore junctions. Langmuir 2015, 31(8): 2600-2608.

Shou, D., Ye, L., Fan, J., et al. Optimal design of porous structures for the fastest liquid absorption. Langmuir 2014a, 30(1): 149-155.

Shou, D., Ye, L., Fan, J., et al. Geometry-induced asymmetric capillary flow. Langmuir 2014b, 30(19): 5448-5454.

Tsunazawa, Y., Yokoyama, T., Nishiyama, N. An experimental study on the rate and mechanism of capillary rise in sandstone. Prog. Earth Planet. Sc. 2016, 3(1): 8.

Washburn, E.W. The dynamics of capillary flow. Phys. Rev. 1921, 17(3): 273-283.

Wheeler, T.D., Stroock, A.D. The transpiration of water at negative pressures in a synthetic tree. Nature 2008, 455(7210): 208-212.

Xiao, J., Cai, J., Xu, J. Saturated imbibition under the influence of gravity and geometry. J. Colloid Interf. Sci. 2018, 521: 226-231.

Xiao, J., Stone, H.A., Attinger, D. Source-like solution for radial imbibition into a homogeneous semi-infinite porous medium. Langmuir 2012, 28(9): 4208-4212.

Yue, P., Feng, J.J., Liu, C., et al. A diffuse-interface method for simulating two-phase flows of complex fluids. J. Fluid Mech. 2004, 515: 293-317.

Zhou, C., Yue, P., Feng, J.J. 3D phase-field simulations of interfacial dynamics in newtonian and viscoelastic fluids. J. Comput. Phys. 2010, 229(2): 498-511. 


\section{Appendix}

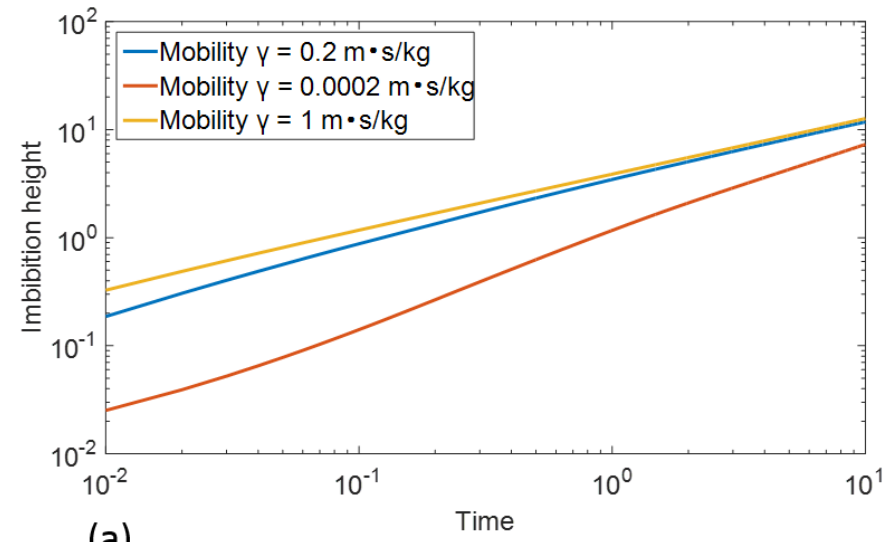

(a)

The meniscus at $\mathrm{t}=0.2 \mathrm{~s}, \gamma=0.2 \mathrm{~m} \cdot \mathrm{s} / \mathrm{kg}$

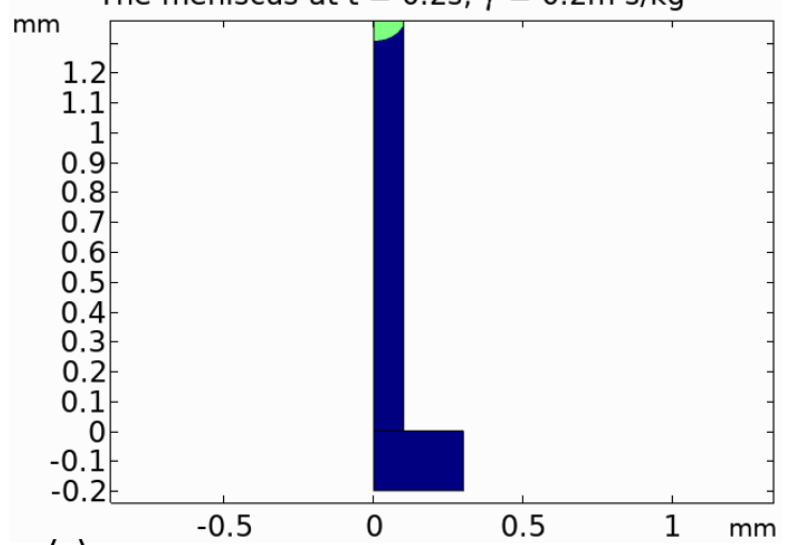

(c)
The meniscus at $\mathrm{t}=0.2 \mathrm{~s}, \gamma=0.0002 \mathrm{~m} \cdot \mathrm{s} / \mathrm{kg}$

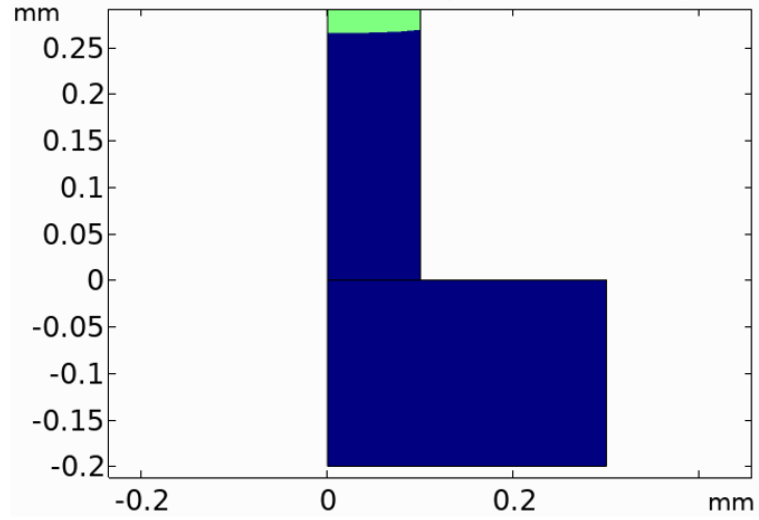

(b)

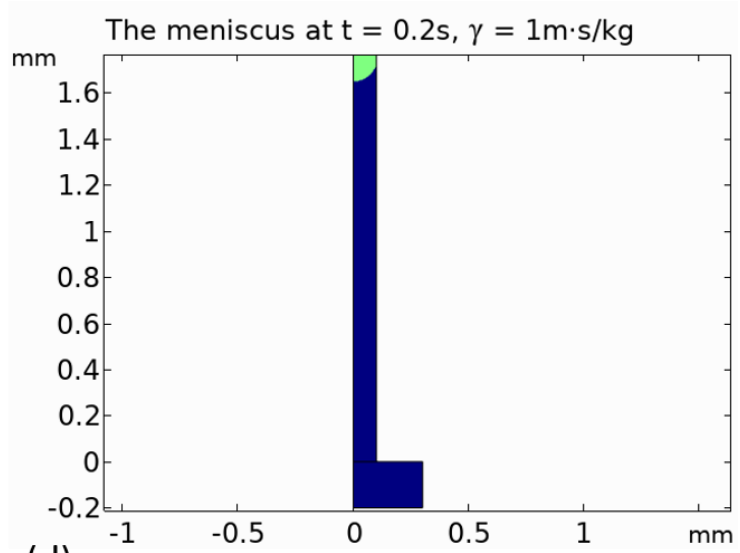

(d)

Fig. S. (a) Imbibition height curves corresponding to different mobility parameters; (b) the meniscus shape and position when $t=0.2 \mathrm{~s}$ and $\gamma=0.0002 \mathrm{~m} \cdot \mathrm{s} / \mathrm{kg}$; (c) the meniscus shape and position when $t=0.2 \mathrm{~s}$ and $\gamma=0.2 \mathrm{~m} \cdot \mathrm{s} / \mathrm{kg} ;(\mathrm{d})$ the meniscus shape and position when $t=0.2 \mathrm{~s}$ and $\gamma=1 \mathrm{~m} \cdot \mathrm{s} / \mathrm{kg}$. 